\title{
Mobility Performance in Community-Dwelling Older Adults: Potential Digital Biomarkers of Concern about Falling
}

\author{
Changhong Wang $^{\text {a }}$ Michelle Patriquin ${ }^{\text {b, d, e }}$ Ashkan Vaziric Bijan Najafi ${ }^{\mathrm{c}}$
}

aDivision of Vascular Surgery and Endovascular Therapy, Department of Surgery, Baylor College of Medicine, Houston, TX, USA; ${ }^{b}$ The Menniger Clinic, Houston, TX, USA; ${ }^{C}$ The BioSensics LLC, Boston, MA, USA; ${ }^{\mathrm{d}}$ Menninger Department of Psychiatry and Behavioral Sciences, Baylor College of Medicine, Houston, TX, USA; ${ }^{\mathrm{C}}$ Michael E. Debakey VA Medical Center, Houston, TX, USA

\section{Keywords}

Mobility performance $\cdot$ Concern about falling $\cdot$ Digital biomarker - Wearables $\cdot$ Remote patient monitoring

\begin{abstract}
Introduction: Concern about falling is a prevalent worry among community-dwelling older adults and may contribute to a decline in physical and mental health. This study aimed to examine the association between mobility performance and concern about falling. Methods: Older adults aged 65 years and older, with Mini-Mental State Examination score $\geq 24$, and ambulatory (with or without the assistive device) were included. Concern about falling was evaluated with Falls Efficacy Scale-International (FES-I) scores. Participants with high concern about falling were identified using the cutoff of FES-I $\geq 23$. Participants' motor capacity was assessed in standardized walking tests under single- and dualtask conditions. Participants' mobility performance was measured based on a 48-h trunk accelerometry signal from a wearable pendant sensor. Results: No significant differences were observed at participant characteristics across groups with different levels of concern about falling (low: $N=64$, age $=76.3 \pm 7.2$ years, female $=46 \%$; high: $N=59$, age $=79.3$ \pm 9.1 years, female $=47 \%$ ), after propensity matching with
\end{abstract}

$\mathrm{BMI}$, age, depression, and cognition. With adjustment of motor capacity (stride velocity and stride length under singleand dual-task walking conditions), participants with high concern about falling had significantly poorer mobility performance than those with low concern about falling, including lower walking quantity (walking bouts, steps and time per day, and walking bout average, walking bout variability, and longest walking bout, $p \leq 0.013$ ), and poorer daily-life gait (stride velocity and gait variability, $p \leq 0.023$ ), and poorer walking quality (frontal gait symmetry, and trunk acceleration and velocity intensity, $p \leq 0.041)$. The selected mobility performance metrics (daily steps and frontal gait symmetry) could significantly contribute to identifying older adults with high concern about falling ( $p \leq 0.042$ ), having better model performance $(p=0.036)$ than only walking quantity (daily steps) with adjustment of confounding effects from the motor capacity (stride length under dual-task walking condition). Conclusion: There is an association between mobility performance and concern about falling in older adults. Mobility performance metrics can serve as predictors to identify older adults with high concern about falling, potentially providing digital biomarkers for clinicians to remotely track older adults' change of concern about falling via applications of remote patient monitoring.

다 2021 S. Karger AG, Basel karger@karger.com

www.karger.com/ger

Karger
(C) 2021 S. Karger AG, Basel

(n)
Bijan Najafi

Baylor College of Medicine, Interdisciplinary Consortium on Advanced Motion Performance (iCAMP)

One Baylor Plaza MS:BCM390, Houston, TX 77030 (USA)

bijan.najafi@bcm.edu 


\section{Introduction}

Concern regarding falling is highly prevalent in community-dwelling older adults, with the prevalence rate estimated between 20.8 and $85 \%$ [1-3]. When someone has significant concern about falling, it can lead to a decline in physical and mental performance [1], avoidance of physical activity and social participation [4], increased depression and psychological distress [5], increased physical frailty [4], increased fall risk $[4,6]$, and reduced quality of life [7]. These adverse consequences may lead to more functional impairment and ultimately loss of independence [1].

By assessing for concern about falling in older adults, it can facilitate the recommendation of early intervention strategies [8]. Concern about falling can be evaluated by Falls Efficacy Scale-International (FES-I) or Activitiesspecific Balance and Confidence scale (ABC) $[1,9]$. These scales rely on self-report data and therefore are subjective $[10,11]$. However, there may be more objective correlates of concern about falling. Previous studies have demonstrated that concern about falling is associated with motor capacity metrics measured under standardized walking conditions in a laboratory environment [12-14]. Additionally, self-reported or sensor-measured physical activity restriction is observed in older adults who present with high concern about falling [14-16].

Recent advances in the development of wearable devices allow for the remote monitoring of 'mobility performance' $[17,18]$. Mobility performance refers to walking characteristics under a real-life condition in dynamic home and community environments. Mobility performance has already been identified as a new measure of fall risk $[19,20]$ and cognitive impairment $[21,22]$. Compared with motor capacity, mobility performance can be defined more broadly, including walking quantity (e.g., total steps and bouts per day), daily-life gait (e.g., stride velocity and cadence), and walking quality (e.g., symmetry, intensity, and complexity) $[17,23]$. However, the relationship between mobility performance and concern about falling.is unknown at present.

As such, the current study investigated the relations between mobility performance metrics measured by a wearable pendant motion sensor and concern about falling of older adults. We hypothesized that older adults with high concern about falling would have lower walking quantity, poorer daily-life gait, and reduced walking quality than those with lower concern about falling. We also examined which mobility performance metrics would be independent digital biomarkers that could predict high concern about falling in older adults.

\section{Methods}

\section{Participants}

This study is a secondary analysis of baseline data collected within 2 larger longitudinal studies. The data were from 2 registered studies with ClinicalTrials.gov, identifier NCT01880229 and NCT02819011. These studies were conducted among communitydwelling older adults aged 65 years or older. The first study assessed a person's risk of falling and frailty levels objectively based on physical activity data measured by a wearable sensor. The second study evaluated the long-term effects of bilateral customized ankle-foot orthoses in reducing falling risk in older adults. In the current study, only the baseline measurements (participant characteristics and metrics of motor capacity and mobility performance) were included. Across both studies, participants were recruited from primary, secondary, and tertiary health care settings, community providers, assisted living facilities, retirement homes, and aging service organizations. Participant inclusion criteria included being able to walk a distance of at least $1.8 \mathrm{~m}$ ( 6 feet $)$ without assistance and the use of walking-assistive devices (such as canes) in daily living allowed. Exclusion criteria included a MiniMental State Examination (MMSE) score $\leq 23$, terminal illness, and unwillingness to participate. Eligible subjects signed a written informed consent form, approved by local institutional review boards (University of Arizona and Baylor College of Medicine).

\section{Outcome Measures}

Participant Characteristics

Participants were interviewed to collect demographic and health information (age, sex, and BMI calculated from height and weight). Participants' depressive symptoms, concern about falling, and cognitive performance were assessed using the Center for Epidemiologic Studies Depression Scale (CES-D) [24], the FES-I [25], and the MMSE [26], respectively. Participants with high concern about falling were categorized based on the cutoff threshold of FES-I $\geq 23$ [9].

\section{Motor Capacity}

Participants' motor capacity was assessed under single- and dual-task (counting backward by 1, starting from 100) walking conditions under supervision of a trained examiner. Participants walked $4.57 \mathrm{~m}$ (15 feet) at a self-selected speed under each condition. Spatial-temporal gait metrics (stride velocity, stride time, stride length, and coefficient of variation [CV] of stride velocity) were measured using shank- and waist-attached wearable sensors (LEGSys $^{\mathrm{TM}}$, Biosensics, Boston, MA, USA) and gait analysis algorithms which have been described previously in details [27].

\section{Mobility Performance}

Triaxial accelerometry signals were collected continuously over 48 h using a pendant motion sensor (PAMSys ${ }^{\mathrm{TM}}$, Biosensics, Boston, MA, USA). Participants were encouraged to wear it for all time, except while showering. Unbroken walking bouts were detected using the analysis algorithm developed by Najafi et al. [28, 29]. The algorithm has been validated in both older adults with and without using walking-assistive devices (such as a cane) [28]. Metrics of walking quantity were extracted from walking bouts with at least 3 successive steps $[28,30]$, including the number of walking bouts and steps per day, and walking time (as percentage of total monitoring time), as well as the mean, standard deviation (SD), 
Table 1. Mobility performance metrics and their definitions

\begin{tabular}{|c|c|}
\hline Mobility performance metrics & Definitions \\
\hline \multicolumn{2}{|l|}{ Walking quantity } \\
\hline Daily walking bouts, $n$ & $\begin{array}{l}\text { Number of walking bouts over } 24 \mathrm{~h} \text {. A walking bout was defined by at least } \\
3 \text { consecutive steps as suggested by Najafi et al. [28] }\end{array}$ \\
\hline Daily steps, $n$ & Number of walking steps over $24 \mathrm{~h}$ \\
\hline Daily walking time, $\%$ & Time spent in walking as percentage of $24 \mathrm{~h}$ \\
\hline Walking bout average, $n$ & Mean of step counts of all walking bouts \\
\hline Longest walking bout, $n$ & Maximum of step counts of all walking bouts \\
\hline \multicolumn{2}{|l|}{ Daily-life gait } \\
\hline Stride time, s & Mean of stride time of all walking bouts \\
\hline Stride length, m & Mean of stride lengths of all walking bouts \\
\hline Stride velocity, $\mathrm{m} / \mathrm{s}$ & Mean of stride velocities of all walking bouts \\
\hline Gait unsteadiness, \% & Mean of CVs of stride velocities of all walking bouts \\
\hline Lateral gait symmetry & Harmonic ratio of lateral trunk acceleration \\
\hline Trunk acceleration intensity, $\mathrm{m} / \mathrm{s}^{2}$ & RMS of vertical trunk acceleration \\
\hline Trunk velocity intensity, $\mathrm{m} / \mathrm{s}$ & RMS of vertical trunk velocity \\
\hline Trunk displacement intensity, $\mathrm{cm}$ & RMS of vertical trunk displacement \\
\hline
\end{tabular}

and maximum of step counts across walking bouts. These walking quantity metrics can represent the physical activity level [31]. Metrics of daily-life gait were extracted from walking bouts with at least 10 steps $[29,32]$, including the means of stride time, step length, stride velocity, and CV of stride velocity across walking bouts, as well as the SD of stride velocity across walking bouts. The SD of stride velocity can represent between-walk adaptability [30]. Metrics of walking quality were extracted from the same walking bouts with at least 10 steps, including the means of harmonic ratios in vertical, frontal, and lateral directions across walking bouts, and the means of root mean square of trunk acceleration, velocity, and displacement in the vertical direction across walking bouts. Harmonic ratios are an index of gait symmetry [33], and the root mean square of the trunk vertical motion can represent gait intensity [34]. In these mobility performance metrics, walking quantity and daily-life gait metrics are direct outputs of the assessment device (e.g., daily walking time and walking steps, and stride velocity), using validated algorithms described in our previous studies [29,35], or simple descriptive statistics of these outputs (e.g., the mean, SD, and maximum of steps across walking bouts, and the SD of stride velocity across walking bouts). Differently, walking quality metrics (e.g., harmonic ratio) were calculated from raw accelerometer data using well established signal quantification formulas [33, 34]. Table 1 shows names and definitions of all used mobility performance metrics.

\section{Statistical Analyses}

All statistical analyses were performed using IBM SPSS Statistics version 24 (IBM, Armonk, NY, USA). For all statistical analyses, significance was accepted at $p<0.05$. Participant characteris- tics across levels of concern about falling (binary: high and low) were compared using one-way ANOVA for continuous variables that are normally distributed, or using Mann-Whitney $U$ test for continuous variables that are not normally distributed. Normality was checked using the Shapiro-Wilk test $(p>0.05)$. Additionally, $\chi^{2}$ test was used to analyze between-group differences for categorical variables.

To more robustly evaluate the influence of concern about falling on mobility performance, propensity score matching was used to generate a matched case-control subsample, reducing confounding effects from participant characteristics except for the grouping factor (FES-I scores) [36, 37]. Participant characteristics that showed significant differences across levels of concern about falling in the total sample before the matching were selected as covariates for the propensity matching. The match tolerance was set to 0.2 . The matching procedure ran a logistic regression on the group indicator (levels of concern about falling), and then used the resulting propensity variable to select controls for the cases without replacement.

Then, based on the post-matched subsample, ANOVA was used to analyze between-group differences for motor capacity and mobility performance metrics. For mobility performance metrics, additional analysis of covariance (ANCOVA) was used to analyze between-group differences for mobility performance metrics again, with adjustment of the motor capacity metrics that showed significant between-group differences in the last step of the ANOVA. Moreover, the effect size (Cohen's $d$ ) was calculated for each metric of motor capacity and mobility performance.

We used hierarchical multiple logistic regression models to identify older participants with high concern about falling. Only 
Table 2. Participant characteristics before and after propensity score matching

\begin{tabular}{lccr}
\hline $\begin{array}{l}\text { Non-propensity score- } \\
\text { matched groups }\end{array}$ & $\begin{array}{c}\text { Low concern } \\
\text { about falling }\end{array}$ & $\begin{array}{l}\text { High concern } \\
\text { about falling }\end{array}$ & $p$ value \\
\hline$N$ & 63 & 92 & \\
Age, years & $76.3 \pm 7.2$ & $79.6 \pm 8.8$ & 0.036 \\
Female, $n(\%)$ & $46(73)$ & $75(82)$ & 0.209 \\
BMI & $26.9 \pm 5.4$ & $29.0 \pm 6.4$ & 0.043 \\
CES-D & $5.9 \pm 5.7$ & $10.0 \pm 7.4$ & 0.001 \\
MMSE & $29.1 \pm 1.3$ & $28.5 \pm 1.6$ & 0.042 \\
FES-I & $19.1 \pm 1.8$ & $34.9 \pm 10.4$ & $<0.001$ \\
Propensity score-matched groups & & \\
$\quad$ Number & 63 & 59 & \\
$\quad$ Age, years & $76.3 \pm 7.2$ & $79.3 \pm 9.1$ & 0.091 \\
Female, $n(\%)$ & $46(73)$ & $47(80)$ & 0.389 \\
BMI & $26.9 \pm 5.4$ & $28.3 \pm 6.3$ & 0.292 \\
CES-D & $5.9 \pm 5.7$ & $7.5 \pm 6.4$ & 0.141 \\
MMSE & $29.1 \pm 1.3$ & $28.9 \pm 1.3$ & 0.310 \\
FES-I & $19.1 \pm 1.8$ & $32.0 \pm 7.7$ & $<0.001$ \\
\hline
\end{tabular}

CES-D, Center for Epidemiologic Studies Depression Scale; MMSE, Mini-Mental State Examination; FES-I, Fall Efficacy ScaleInternational.

the motor capacity and mobility performance metrics that showed significant between-group differences via ANCOVA analyses were considered as predictors. The logistic model includes 3 blocks: Motor capacity metrics were inputted into the first block, as the control of confounding factors. Then, mobility performance metrics were inputted sequentially, where walking quantity metrics were inputted into the second block as a benchmark, and daily-life gait and walking quality metrics were inputted into the third block. The absence of multicollinearity between the predictors was determined by examining the variance inflation factor (VIF). When 2 or more correlated predictors had VIFs higher than 10 , the 1 with the lowest effect size would be removed until all predictors' VIFs were below 10. For each block of this multiple logistic regression model, feature selection of the backward stepwise LR method was used. The model performance (Nagelkerke $R^{2}$, accuracy, change in $\chi^{2}$ and significance ( $p$ value), area under the curve from receiver operating characteristics analysis), and each predictor's coefficient (B) and significance ( $p$ value) were reported.

\section{Results}

A total of 155 older adults satisfied inclusion and exclusion criteria and were included in this study. The total sample was stratified based on FES-I score into 2 groups with low concern about falling $(N=63)$ and high concern about falling $(N=92)$. There were significant group (low vs. high concern about falling) differences observed in age, BMI, CES-D, MMSE, and FES-I scores. After pro-
Table 3. Motor capacity in participants across levels of concern about falling

\begin{tabular}{lcccc}
\hline & $\begin{array}{l}\text { Low concern } \\
\text { about falling } \\
(N=63)\end{array}$ & $\begin{array}{l}\text { High } \\
\text { concern } \\
\text { about falling } \\
(N=59)\end{array}$ & $p$ value & $\begin{array}{l}\text { Effect } \\
\text { size }\end{array}$ \\
& & & & \\
\hline Single-task walking & $1.11 \pm 0.19$ & $1.00 \pm 0.29$ & 0.024 & 0.45 \\
$\quad$ Stride velocity, m/s & $5.3 \pm 3.4$ & $5.9 \pm 3.6$ & 0.173 & 0.17 \\
CV of stride velocity, \% & $1.14 \pm 0.13$ & $1.19 \pm 0.18$ & 0.064 & 0.32 \\
$\quad$ Stride time, $\mathrm{s}$ & $1.22 \pm 0.15$ & $1.14 \pm 0.23$ & 0.029 & 0.41 \\
$\quad$ Stride length, m & & & & \\
Dual-task walking & $0.99 \pm 0.23$ & $0.89 \pm 0.29$ & 0.049 & 0.38 \\
$\quad$ Stride velocity, m/s & $7.0 \pm 3.3$ & $7.9 \pm 3.8$ & 0.225 & 0.25 \\
CV of stride velocity, \% & $1.30 \pm 0.28$ & $1.33 \pm 0.26$ & 0.352 & 0.11 \\
$\quad$ Stride time, $\mathrm{s}$ & $1.22 \pm 0.16$ & $1.12 \pm 0.24$ & 0.009 & 0.49 \\
$\quad$ Stride length, m & & & & \\
\hline
\end{tabular}

$\mathrm{CV}$, coefficients of variation. $p$ value is calculated from ANOVA; effect size is calculated using Cohen's $d$.

pensity score matching with age, BMI, CES-D, and MMSE scores, 122 participants (63 participants with low concern about falling and 59 participants with high concern about falling) were retained for the subsequent analyses. Significant differences were no longer observed in BMI, CES-D, and MMSE scores after propensity score matching. Table 2 reports participant characteristics before and after propensity score matching.

Table 3 shows differences between groups (high vs. low concern about falling) for motor capacity. Significant differences were observed for stride velocity and stride length under single- and dual-task walking conditions.

Table 4 shows differences between groups (high vs. low concern about falling) for mobility performance metrics. Significant between-group differences were observed for all metrics in walking quantity, for stride length, stride velocity, and gait variability in daily-life gait, and for vertical and frontal gait symmetry, intensity of trunk acceleration, velocity, and displacement in walking quality. After adjustment with motor capacity metrics (stride velocity and stride length under both single- and dual-task conditions), between-group differences remained statistically significant for all metrics in walking quantity, for stride velocity and gait variability in daily-life gait, and for frontal gait symmetry and intensity of trunk acceleration and velocity in walking quality.

Table 5 shows predictors and model performance of the hierarchical logistic regression analysis. When only entering block 1 (motor capacity metrics), the metric of stride length under dual-task walking condition was se- 
Table 4. Mobility performance of participants across levels of concern about falling

\begin{tabular}{|c|c|c|c|c|c|}
\hline & $\begin{array}{l}\text { Low concern } \\
\text { about falling } \\
(N=63)\end{array}$ & $\begin{array}{l}\text { High concern } \\
\text { about falling } \\
(N=59)\end{array}$ & $p$ value $^{1}$ & $p$ value $^{2}$ & $\begin{array}{l}\text { Effect } \\
\text { size }\end{array}$ \\
\hline \multicolumn{6}{|l|}{ Walking quantity } \\
\hline Daily walking bouts, $n$ & $183.8 \pm 78.1$ & $125.9 \pm 68.9$ & $<0.001$ & $<0.001$ & 0.79 \\
\hline Daily steps, $n$ & $4,745.0 \pm 2,432.4$ & $2,726.7 \pm 1,639.0$ & $<0.001$ & $<0.001$ & 0.97 \\
\hline Daily walking time, $\%$ & $7.0 \pm 3.9$ & $3.9 \pm 2.6$ & $<0.001$ & $<0.001$ & 0.94 \\
\hline Walking bout average, $n$ & $32.3 \pm 11.7$ & $26.3 \pm 8.0$ & 0.001 & 0.004 & 0.97 \\
\hline Walking bout variability, $n$ & $73.7 \pm 68.6$ & $36.2 \pm 37.9$ & 0.002 & 0.001 & 0.68 \\
\hline Longest walking bout, $n$ & $948.9 \pm 1,050.7$ & $449.8 \pm 810.1$ & 0.004 & 0.013 & 0.53 \\
\hline \multicolumn{6}{|l|}{ Daily-life gait } \\
\hline Stride time, s & $1.64 \pm 0.15$ & $1.66 \pm 0.15$ & 0.520 & 0.583 & 0.13 \\
\hline Stride length, m & $1.36 \pm 0.16$ & $1.27 \pm 0.19$ & 0.009 & 0.123 & 0.51 \\
\hline Stride velocity, $\mathrm{m} / \mathrm{s}$ & $0.95 \pm 0.12$ & $0.87 \pm 0.13$ & 0.001 & 0.023 & 0.64 \\
\hline Gait unsteadiness, $\%$ & $40.8 \pm 5.8$ & $41.7 \pm 5.3$ & 0.384 & 0.944 & 0.16 \\
\hline Gait variability, m/s & $0.23 \pm 0.05$ & $0.19 \pm 0.05$ & $<0.001$ & 0.005 & 0.80 \\
\hline \multicolumn{6}{|l|}{ Walking quality } \\
\hline Vertical gait symmetry & $1.65 \pm 0.25$ & $1.55 \pm 0.24$ & 0.032 & 0.100 & 0.41 \\
\hline Frontal gait symmetry & $1.53 \pm 0.25$ & $1.42 \pm 0.20$ & 0.008 & 0.041 & 0.49 \\
\hline Lateral gait symmetry & $1.73 \pm 0.44$ & $1.69 \pm 0.31$ & 0.820 & 0.309 & 0.11 \\
\hline Trunk acceleration intensity, $\mathrm{m} / \mathrm{s}^{2}$ & $1.03 \pm 0.17$ & $0.92 \pm 0.16$ & 0.002 & 0.011 & 0.67 \\
\hline Trunk velocity intensity, $\mathrm{m} / \mathrm{s}$ & $0.08 \pm 0.01$ & $0.07 \pm 0.01$ & 0.002 & 0.017 & 1.00 \\
\hline Trunk displacement intensity, $\mathrm{cm}$ & $1.18 \pm 0.23$ & $1.08 \pm 0.20$ & 0.010 & 0.057 & 0.46 \\
\hline
\end{tabular}

$p$ value $^{1}$ is calculated from ANOVA, analysis without adjustment; $p$ value ${ }^{2}$ is calculated from ANCOVA, analysis with adjustment of the selected motor capacity metrics (stride velocity and stride length under both single- and dual-task conditions); effect size is calculated using Cohen's $d$.

Table 5. Logistic regression results to identify participants with high concern about falling

\begin{tabular}{|c|c|c|c|c|c|}
\hline & \multicolumn{2}{|l|}{ Predictors } & \multicolumn{3}{|c|}{ Results } \\
\hline \multirow{2}{*}{$\begin{array}{l}\text { Blocks } 1+2 \text { : Motor capacity + walking } \\
\text { quantity }\end{array}$} & DTW stride length & $-1.106(0.318)$ & 0.297 & 66.9 & $21.556(<0.001)$ \\
\hline & Daily steps & $-0.001(<0.001)$ & & & \\
\hline \multirow{2}{*}{$\begin{array}{l}\text { Blocks } 1+2+3 \text { : Motor capacity }+ \text { walking } \\
\text { quantity }+ \text { daily-life gait }+ \text { walking quality }\end{array}$} & Daily steps & $-0.001(<0.001)$ & & & \\
\hline & Frontal gait symmetry & $-2.003(0.042)$ & & & \\
\hline
\end{tabular}

DTW, dual-task walking. B: coefficients of determinants. $R^{2}$ represents percentage of the explained variability. Accuracy represents classification ability of the model to identify those with the high concern about falling. $\Delta \chi^{2}$ represents the change of the model performance as a new block is added into the model, where the $\Delta \chi^{2}$ for the model with only block 1 (the first data row) represents the improvement between the model with block 1 and the model without only constant.

lected as an independent predictor $(p=0.002)$, and the model accounted for $9.0 \%$ of the explained variability $\left(R^{2}=0.090\right)$ with the accuracy of $62.7 \%$. When further entering block 2 (walking quantity metrics), the metric of daily steps was added into the model as an independent predictor $(p<0.001)$, but at this time, the metric of stride length under dual-task walking condition was no longer the significant predictor $(p=0.318)$. The model with 
blocks 1 and 2 accounted for $29.7 \%$ of the explained variability $\left(R^{2}=0.297\right)$ with an accuracy of $66.9 \%$. Compared with the model with only block 1 , the model with blocks 1 and 2 identified the group with high concern about falling significantly better $\left(\chi^{2}=21.556, p<0.001\right)$. Finally, after entering block 3 (daily-life gait and walking quality metrics), the metric of frontal gait symmetry was added into the model as an independent predictor $(p=0.042)$, and the metric of daily steps was still an independent predictor $(p<0.001)$, while the metric of stride length under dual-task walking condition was not the independent predictor $(p=0.501)$. Compared with the model with blocks 1 and 2, the model with all 3 blocks identified the group with high concern about falling significantly better $\left(\chi^{2}=4.378, p=0.036\right)$. Figure 1 shows the receiver operating characteristics curves of the logistic regression model. By sequentially adding the blocks into the model, the area under the curve increased from 0.644 , to 0.747 and to 0.772 .

\section{Discussion}

Our results indicate that older adults who present with high concern about falling demonstrate: lower walking quantity (daily walking bouts, daily steps, daily walking time), poorer daily-life gait (stride velocity, gait variability), and poorer walking quality (frontal gait symmetry, trunk acceleration and velocity intensity), after controlling for participant characteristics (age, BMI, CES-D, and MMSE) and motor capacity (stride velocity and stride length under both single- and dual-task walking conditions). We also found that selected mobility performance metrics (daily steps and frontal gait symmetry) were significant predictors of the group with high concern about falling after controlling for motor capacity metrics (stride length of dual-task walking), indicating that these mobility performance metrics may be useful to triage objectively older adults with high concern about falling.

Notably, our participants with high concern about falling were significantly older and had increased depression, more cognitive impairment, and greater BMI relative to those with fewer falling concerns (Table 2). These findings are congruent with the epidemiological studies [7, 38-40]. Additionally, significant reductions were found in motor capacity metrics (stride velocity, stride length under single- and dual-task walking conditions) in older participants with high concern about falling, compared with those with low concern about falling (Table 3 ). This

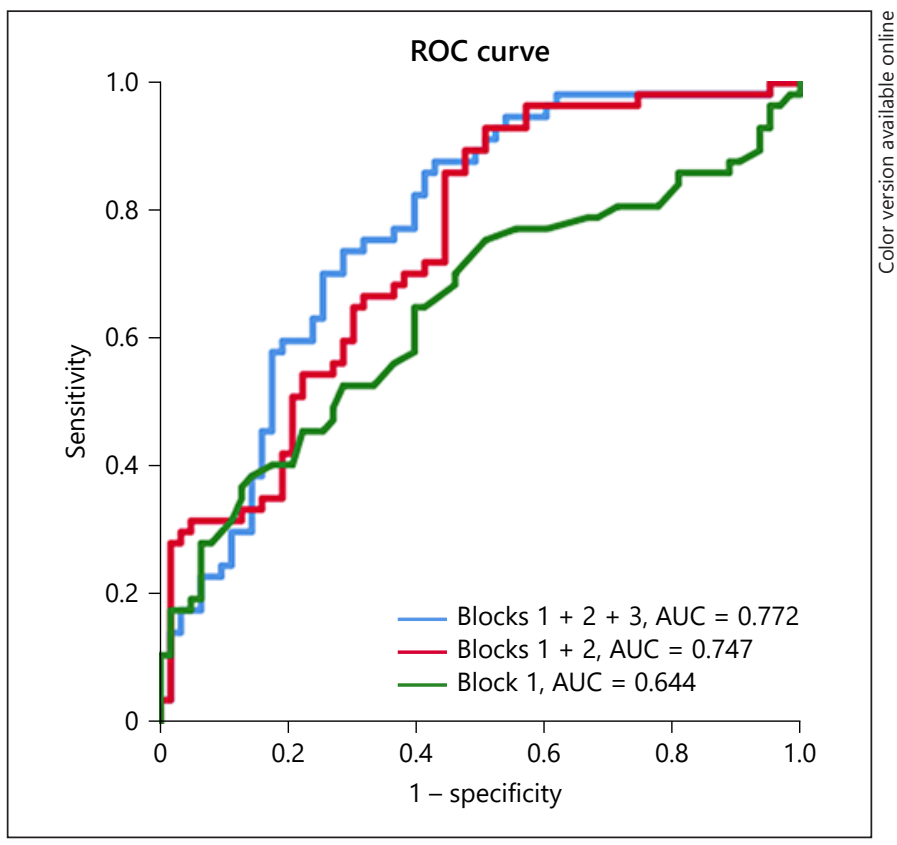

Fig. 1. ROC curves for the hierarchical logistic regression model with the 3 blocks (motor capacity, walking quantity, and habitual gait and walking quality). The AUC values are $0.644,0.747$, and 0.772 for different levels of this model. ROC, receiver operating characteristics; AUC, area under the curve.

finding indicates a potential cognitive influence on walking capacity (e.g., fear of falling and anxiety about falling), which is in line with previous studies [12-14].

More importantly, this is the first study to demonstrate the influence of concern about falling on mobility performance, including not only walking quantity but also habitual gait and walking quality. After adjustment for motor capacity (stride velocity and stride length in single- and dual-task standardized walking), some mobility performance metrics (such as daily walking bouts, steps, and walking time, walking bout average, walking bout variability, longest walking bout, stride velocity, gait variability, frontal gait symmetry, and trunk acceleration and velocity intensity) still showed significant differences across groups with different levels of concern about falling (Table 4). The results reflect the adverse influence of concern about falling on daily walking behavior irrespective of walking capacity. This finding provides a novel insight into influence of concern about falling to daily walking activity, extending from traditional physical activity restriction [14-16]. Additionally, these impaired mobility performance metrics were found to be associated with retrospective and prospective falls in previous studies $[19,20,34]$ and may play a 
role as mediators between concern about falling and prospective falls.

Few studies investigated sensor-derived physical activity (walking quantity) across levels of concern about falling. Paraschiv-Ionescu et al. [41] reported that there were no significant differences across levels of concern about falling for time spent for walking, steps of the longest walking bout (max steps of all walking bouts), and average cadence (an inverse form of stride time). These findings do not match consensus obtained from the known questionnaire-based studies $[2,42]$ and it may because of underpowered statistical comparison on relatively small sample size $(N=40)$. In contrast, we observed significant between-group differences in walking quantity (see Table 4) based on our post-matched subsample with more than 100 participants. The reduction in walking quantity in participants with high concern about falling may indicate fear-related avoidance of healthy lifestyles, such as outdoor walking, leisure-time physical activity, and access to recreational or community facilities.

The final prediction model for high concern about falling comprised only 2 mobility performance metrics (daily steps and frontal gait symmetry) as significant predictors with 1 motor capacity metric (stride length under dual-task walking condition) as a covariate (Table 5). None of the daily-life gait metrics were included in the final model. It may because there is a strong correlation between motor capacity (clinical gait) and daily-life gait. The sign symbols of the 2 independent predictors' coefficients in the final model may indicate that lower daily steps and frontal gait symmetry in mobility performance are associated with an increased likelihood of having a high concern about falling. The results suggest that the selected mobility performance metrics could serve as objective indicators from which to triage the individual with potentially high concern about falling for further in-clinic evaluation or tele-medicine consultation. Compared with direct investigation of concern about falling using the questionnaire (e.g., FES-I), the sensor-based mobility performance metrics may provide a chance to track change of concern about falling, even before the individual has an insight into the subtle change.

One limitation of our study on the technical side is that the mobility performance metrics were derived from 48 - $h$ continuous accelerometry data recording, which may not cover the day-to-day variability of physical activity patterns among community-dwelling older adults. There is still controversy about the minimum number of days re-

Mobility Performance as Digital

Biomarkers of Concern about Falling quired for assessing mobility performance. The periods of previous studies varied from 2 to 14 days $[23,41]$. In the future, we need to investigate whether the day-to-day variability of mobility performance will influence the identification of levels of concern about falling for our applications.

Several limitations were also generated from our sample. Besides the limited sample size, the generalizability of our results to the general older population may be limited by the fact that only cognitive-intact older people were included in this study. A social desirability bias (a psychological influence that the participant knows a sensor monitors him/her) may cause the older participants to have higher physical activity level than a normal condition. Additionally, we must acknowledge that propensity score matching used in the current analyses could not entirely exclude the other potential confounding factors which we did not measure. Future studies are needed to verify whether the association between mobility performance and concern about falling still exists in a larger sample across various patient populations (e.g., cognitive-impaired, or those with different comorbidities) within improved study designs (e.g., prospective observational studies, or longitudinal studies). Additionally, practicability, acceptance, and adherence to wearing the sensor would also have to be considered and investigated.

In conclusion, there is a significant association between mobility performance and concern about falling. The selected mobility performance metrics can serve as predictors to identify older adults with high concern about falling, potentially providing digital biomarkers for clinicians to remotely track older adults' change in levels of concern about falling in applications of remote patient monitoring.

\section{Acknowledgements}

The authors would like to acknowledge Ms. Maria Noun, Ms. Sogol Golafshan, and Ms. Sanam Sharafkhaneh who contributed to data management. The authors also would like to give special thanks to Ms. Qianzi Zhang for her support on statistical analyses.

\section{Statement of Ethics}

The data were from 2 registered studies with ClinicalTrials.gov, identifier NCT01880229 and NCT02819011. Eligible subjects signed a written informed consent form, approved by local institutional review boards (University of Arizona and Baylor College of Medicine). 


\section{Conflict of Interest Statement}

A.V. is with BioSensics LLC, manufacturer of PAMsys used in this study. He is also serving as the Principal Investigator of a NIH SBIR grant, which partially sponsored this study. He has, however, only contributed in the technical aspect of this study and did not involve in patient recruitment, data analysis, or interpretation of clinical data. None of other co-authors claimed conflict of interest relevant to the scope of this study.

\section{Funding Sources}

This work was supported by the National Institutes of Health/ National Institute on Agings (award numbers SB1AG032748 and R42AG060853). M.P. is supported through funding from The
Menninger Clinic and The Menninger Clinic Foundation. The content is solely the responsibility of the authors and does not necessarily represent the official views of the sponsors.

\section{Author Contributions}

C.W., M.P., and B.N. helped in study design, analysis, and interpretation of data. A.V. provided technical support in interpretation of raw signals from the wearable sensor. C.W. wrote the manuscript. All the authors contributed to revising it critically for important intellectual content and final approval of the version.

\section{References}

1 Scheffer AC, Schuurmans MJ, van Dijk N, van der Hooft T, de Rooij SE. Fear of falling: measurement strategy, prevalence, risk factors and consequences among older persons. Age Ageing. 2008;37(1):19-24.

2 Zijlstra G, van Haastregt JC, van Eijk JT, van Rossum E, Stalenhoef PA, Kempen GI. Prevalence and correlates of fear of falling, and associated avoidance of activity in the general population of community-living older people. Age Ageing. 2007;36(3):3049.

3 Dierking L, Markides K, Al Snih S, Kristen Peek M. Fear of falling in older Mexican Americans: a longitudinal study of incidence and predictive factors. J Am Geriatr Soc. 2016; 64(12):2560-5.

4 Delbaere K, Crombez G, Vanderstraeten G, Willems T, Cambier D. Fear-related avoidance of activities, falls and physical frailty. A prospective community-based cohort study. Age Ageing. 2004;33(4):368-73.

5 van Haastregt JCM, Zijlstra GAR, van Rossum E, van Eijk JTM, Kempen GIJM. Feelings of anxiety and symptoms of depression in community-living older persons who avoid activity for fear of falling. Am J Geriatr Psychiatry. 2008;16(3):186-93.

6 Clemson L, Kendig H, Mackenzie L, Browning C. Predictors of injurious falls and fear of falling differ: an 11-year longitudinal study of incident events in older people. J Aging Health. 2015;27(2):239-56.

7 Arfken CL, Lach HW, Birge SJ, Miller JP. The prevalence and correlates of fear of falling in elderly persons living in the community. Am J Public Health. 1994;84(4):565-70.

8 Zijlstra GA, van Haastregt JC, van Rossum E, van Eijk JT, Yardley L, Kempen GI. Interventions to reduce fear of falling in communityliving older people: a systematic review. J Am Geriatr Soc. 2007;55(4):603-15.
9 Delbaere K, Close JC, Mikolaizak AS, Sachdev PS, Brodaty H, Lord SR. The falls efficacy scale international (FES-I): a comprehensive longitudinal validation study. Age Ageing. 2010; 39(2):210-6.

10 Tinetti ME, Richman D, Powell L. Falls efficacy as a measure of fear of falling. J Gerontol. 1990;45(6):P239-43.

11 Reder P, Lucey C. Assessment of parenting: psychiatric and psychological contributions. Routledge. 2014.

12 Reelick MF, van Iersel MB, Kessels RP, Rikkert MG. The influence of fear of falling on gait and balance in older people. Age Ageing. 2009;38(4):435-40.

13 Rochat S, Büla CJ, Martin E, SeematterBagnoud L, Karmaniola A, Aminian K, et al. What is the relationship between fear of falling and gait in well-functioning older persons aged 65 to 70 years? Arch Phys Med Rehabil. 2010;91(6):879-84.

14 Patil R, Uusi-Rasi K, Kannus P, Karinkanta S, Sievänen $\mathrm{H}$. Concern about falling in older women with a history of falls: associations with health, functional ability, physical activity and quality of life. Gerontology. 2014; 60(1):22-30.

15 Yardley L, Smith H. A prospective study of the relationship between feared consequences of falling and avoidance of activity in community-living older people. Gerontologist. 2002; 42(1):17-23.

16 Jefferis BJ, Iliffe S, Kendrick D, Kerse N, Trost $S$, Lennon LT, et al. How are falls and fear of falling associated with objectively measured physical activity in a cohort of communitydwelling older men? BMC Geriatr. 2014;14: 114.

17 Din SD, Godfrey A, Rochester L. Validation of an accelerometer to quantify a comprehensive battery of gait characteristics in healthy older adults and parkinson's disease: toward clinical and at home use. IEEE J Biomed Health Inform. 2016;20(3):838-47.

18 Brodie MA, Lord SR, Coppens MJ, Annegarn J, Delbaere K. Eight-week remote monitoring using a freely worn device reveals unstable gait patterns in older fallers. IEEE Trans Biomed Eng. 2015;62(11):2588-94.

19 van Schooten KS, Pijnappels M, Rispens SM, Elders PJ, Lips P, van Dieën JH. Ambulatory fall-risk assessment: amount and quality of daily-life gait predict falls in older adults. J Gerontol A Biol Sci Med Sci. 2015;70(5):608-15.

20 Del Din S, Galna B, Godfrey A, Bekkers EMJ, Pelosin E, Nieuwhof F, et al. Analysis of freeliving gait in older adults with and without parkinson's disease and with and without a history of falls: identifying generic and disease-specific characteristics. J Gerontol A Biol Sci Med Sci. 2017;74(4):500-6.

21 Taylor ME, Brodie MA, van Schooten KS, Delbaere K, Close JCT, Payne N, et al. Older people with dementia have reduced daily-life activity and Impaired daily-life gait when compared to age-sex matched controls. J Alzheimers Dis. 2019;71(Suppl 1):S125-35

22 Hausdorff JM, Hillel I, Shustak S, Del Din S, Bekkers EMJ, Pelosin E, et al. Everyday stepping quantity and quality among older adult fallers with and without mild cognitive impairment: initial evidence for new motor markers of cognitive deficits? J Gerontol A Biol Sci Med Sci. 2017;73(8):1078-82.

23 Weiss A, Brozgol M, Dorfman M, Herman T, Shema S, Giladi N, et al. Does the evaluation of gait quality during daily life provide insight into fall risk? a novel approach using 3-day accelerometer recordings. Neurorehabil Neural Repair. 2013;27(8):742-52.

24 Radloff LS. The CES-D scale: a self-report depression scale for research in the general population. Appl Psychol Meas. 1977;1(3):385401. 
25 Yardley L, Beyer N, Hauer K, Kempen G, Piot-Ziegler C, Todd C. Development and initial validation of the falls efficacy scale-international (FES-I). Age Ageing. 2005;34(6): 614-9.

26 Folstein MF, Folstein SE, McHugh PR. "Minimental state": a practical method for grading the cognitive state of patients for the clinician. J Psychiatr Res. 1975;12(3):189-98.

27 Aminian K, Najafi B, Büla C, Leyvraz PF, Robert P. Spatio-temporal parameters of gait measured by an ambulatory system using miniature gyroscopes. J Biomech. 2002;35(5): 689-99.

28 Najafi B, Aminian K, Paraschiv-Ionescu A, Loew F, Büla CJ, Robert P. Ambulatory system for human motion analysis using a kinematic sensor: monitoring of daily physical activity in the elderly. IEEE Trans Biomed Eng. 2003;50(6):711-23.

29 Najafi B, Armstrong DG, Mohler J. Novel wearable technology for assessing spontaneous daily physical activity and risk of falling in older adults with diabetes. J Diabetes Sci Technol. 2013;7(5):1147-60.

30 Brodie MA, Coppens MJ, Ejupi A, Gschwind YJ, Annegarn J, Schoene D, et al. Comparison between clinical gait and daily-life gait assessments of fall risk in older people. Geriatr Gerontol Int. 2017;17(11):2274-82.
31 Jansen CP, Toosizadeh N, Mohler MJ, Najafi B, Wendel C, Schwenk M. The association between motor capacity and mobility performance: frailty as a moderator. Eur Rev Aging Phys Act. 2019;16(1):16.

32 Brodie MA, Coppens MJ, Lord SR, Lovell NH Gschwind YJ, Redmond SJ, et al. Wearable pendant device monitoring using new wavelet-based methods shows daily life and laboratory gaits are different. Med Biol Eng Comput. 2016;54(4):663-74.

33 Bellanca JL, Lowry KA, Vanswearingen JM, Brach JS, Redfern MS. Harmonic ratios: a quantification of step to step symmetry. J Biomech. 2013;46(4):828-31.

34 van Schooten KS, Pijnappels M, Rispens SM, Elders PJ, Lips P, Daffertshofer A, et al. Dailylife gait quality as predictor of falls in older people: a 1-year prospective cohort study. PLos One. 2016;11(7):e0158623.

35 Toosizadeh N, Mohler J, Najafi B. Assessing upper extremity motion: an innovative method to identify frailty. J Am Geriatr Soc. 2015; 63(6):1181-6.

36 Austin PC. An introduction to propensity score methods for reducing the effects of confounding in observational studies. Multivariate Behav Res. 2011;46(3):399-424.

37 Yao XI, Wang X, Speicher PJ, Hwang ES, Cheng P, Harpole DH, et al. Reporting and guidelines in propensity score analysis: a systematic review of cancer and cancer surgical studies. J Natl Cancer Inst. 2017;109(8).

38 van Landingham SW, Massof RW, Chan E, Friedman DS, Ramulu PY. Fear of falling in age-related macular degeneration. BMC Ophthalmol. 2014;14:10.

39 Painter JA, Allison L, Dhingra P, Daughtery J, Cogdill K, Trujillo LG. Fear of falling and its relationship with anxiety, depression, and activity engagement among community-dwelling older adults. Am J Occup Ther. 2012; 66(2):169-76.

40 Uemura K, Shimada H, Makizako H, Doi T, Tsutsumimoto K, Yoshida D, et al. Effects of mild and global cognitive impairment on the prevalence of fear of falling in communitydwelling older adults. Maturitas. 2014;78(1): 62-6.

41 Paraschiv-Ionescu A, Büla CJ, Major K, Lenoble-Hoskovec C, Krief H, El-Moufawad C, et al. Concern about falling and complexity of free-living physical activity patterns in wellfunctioning older adults. Gerontology. 2018; 64(6):603-11.

42 Cumming RG, Salkeld G, Thomas M, Szonyi G. Prospective study of the impact of fear of falling on activities of daily living, SF-36 scores, and nursing home admission. J Gerontol A Biol Sci Med Sci. 2000;55(5):M299-305. 